\title{
Re-imagining the Scientific Visualization Interaction Paradigm
}

\author{
Daniel F. Keefe and Tobias Isenberg
}

\begin{abstract}
Seeing, touching, sketching, exploring—throughout history these fundamental physical activities have defined our world, regularly supporting creativity and scientific discovery. In recent years, powerful scientific visualization tools have emerged but the potential to closely couple these techniques with natural, physical, spatial human-computer interfaces remains largely untapped. To address these issues we outline a research agenda consisting of six major challenges for natural interfaces for visualization. The technological building blocks are now in place to address these challenges to enable an exciting future where natural interfaces powerfully strengthen and expand use of visualizations in science, engineering, art, and humanities.
\end{abstract}

\section{INTRODUCTION}

$\mathrm{T}$ HE ability to picture and interact with concepts in new ways has always been intrinsic to the process of discovery. Muybridge's classic stroboscopic photographs of horses led to the discovery that all four of a horse's hooves leave the ground during a gallop; at the time this hypothesis was called "unsupported transit." Da Vinci's hand-drawn studies of rushing water informed not only his art, but also the science of hydrodynamics. Today, engineers, scientists, and artists routinely rely upon physical models and 3D prototypes - often it is the physical act of touching, rotating, and annotating these models that brings forth new insights. Imagine if all these visual, physical, spatial human activities could take place in a virtual data visualization space, where powerful computational techniques could be combined with natural human interactions and visual communication.

We believe the key to enabling this exciting future for visualization lies in making the graphical visualization tools we build truly fit seamlessly into the workflows of scientists, engineers, and other users. Given the current landscape of computing, we see an exciting logical path to achieve this progress through new research in natural user interfaces for scientific visualization-research that specifically addresses challenges in incorporating new natural means of input and display into the visualization process.

The term natural user interfaces has been used in a variety of contexts recently [10], often referring to the explosion of multi-touch interfaces as made popular by recent phones, tablets, and surface computing. When discussing natural user interfaces, however, we believe it is essential to first be clear about what we mean by the term. In our conception, natural user interfaces imply moving far beyond the simplistic-but surprisingly common-assumption that merely using a touch interface produces "natural interaction." Rather, we conceive of natural user interfaces as welldesigned human-computer interfaces that enable fluid, often modeless, interaction with computers by interpreting direct physical and/or spatial input from potentially several users. This concept contrast the traditional PC-based interfaces that rely on the mouse and the keyboard for input since these are not only indirect forms of input but also focus on a single interacting person. Creating natural interfaces requires us to solve fundamental research problems to enable scientists to more effectively interact with data.

In this paper, we focus specifically on visualization that involves datasets that are inherently two- or threedimensional and their special constraints [4] - as opposed to working with more abstract data, an area for which efforts to leverage natural interaction have also recently gained traction [3, 8]. For scientific visualization applications, an important argument for natural interfaces is that the physical, spatial style of input they enable can often lead to: (1) an interface with low cognitive overhead, enabling the scientist users to focus more attention on visuals and their own hypotheses as they work; and (2) new capabilities to explore complex data using rich inputs that match the richness of data (e.g., see discussion of 3D data selection techniques in Section 3).

Our primary goal in writing this paper is to identify a concrete research agenda to advance natural user interfaces for scientific visualization because we believe it will become one of the most exciting research areas in our field within the next 20 years. Our primary contribution is, therefore, a set of six specific challenges that we identify and explain through examples in Section 3. Before coming to these, we begin by providing some additional context.

\section{Natural User INTERfaces for Visu- alization: PASt Highlights and CuR- RENT TECHNOLOGY}

As we move to closely couple natural user interfaces with scientific visualization, we can draw upon several success stories from the past. Some of the most outstanding examples come from early work at the University of North Carolina at Chapel Hill that combined interactive displays and experimental interfaces with exciting scientific applications, leading eventually to new tools, such as the Nanomanipulator, that have dramatically impacted 
both visualization research and larger scientific community. New display environments, such as the CAVE and Responsive Workbench, have enabled not only new methods for stereoscopic presentation of data but also new interaction paradigms. More recently, interfaces for Scientific Sketching have shown us how scientists and artists can prototype data visualizations in virtual reality using natural sketchbased input [5], and gesture and touch have been used to interact with volumetric scientific data [6].

Chief among recent innovations in interactive technologies that can be applied to visualization problems is certainly the widespread adoption of touch input, facilitating a more direct interaction than previously possible with keyboard and mouse. Although direct-touch interfaces were developed as early as the 1980's (e.g., Buxton et al.'s work), they have only relatively recently become known and available to a large audience-facilitated by the introduction of multi-touch smart phones and other touch-enabled devices. When combined with appropriate displays, this style of input can enable users to interact directly with objects depicted on the display (direct-touch input).

3D camera devices (e.g., Kinect, Leap) and proximity sensors have also advanced recently. These technologies have the potential to facilitate another rich dimension of input in visualization environments-one quite different from current paradigms-because they do not rely on special props such as hand-held devices or reflective markers.

These advances in input technologies have been made within the context of similar exciting advances in display hardware. "3D TVs" now make stereoscopic displays readily available to the public. Recently, we also have seen that multi-touch input has been coupled with displays such that the touch sensing is built right into the display hardware, providing pixel-precise or better input sensing (touch and pen input). Even displays with adjustable haptic feedback are already being researched. A major implication of these developments is that scientists can now use interactive visualization technologies where they have never been able to do so before. Scientists now have the potential to use affordable whiteboard-sized interactive displays together with colleagues, take visualization tools with them on their mobile interactive tablets, and explore visualizations in collaborative multi-display environments, including anything from a traditional CAVE-like setting to a touch-enabled wall display to mobile tablet-sized devices.

Several significant research challenges must be addressed to realize the truly powerful impact we anticipate these interactive technologies can have for scientific visualization.

\section{Six Challenges for Natural Inter- FACES FOR SCIENTIFIC VISUALIZATION}

In this section, we introduce a research agenda organized as six challenges for natural interfaces for scientific visualization. Specifically, we discuss the need to discover new mappings for the fundamental visual data exploration tasks, to create lasting theories of interaction and support toolkits for major scientific application areas, to support

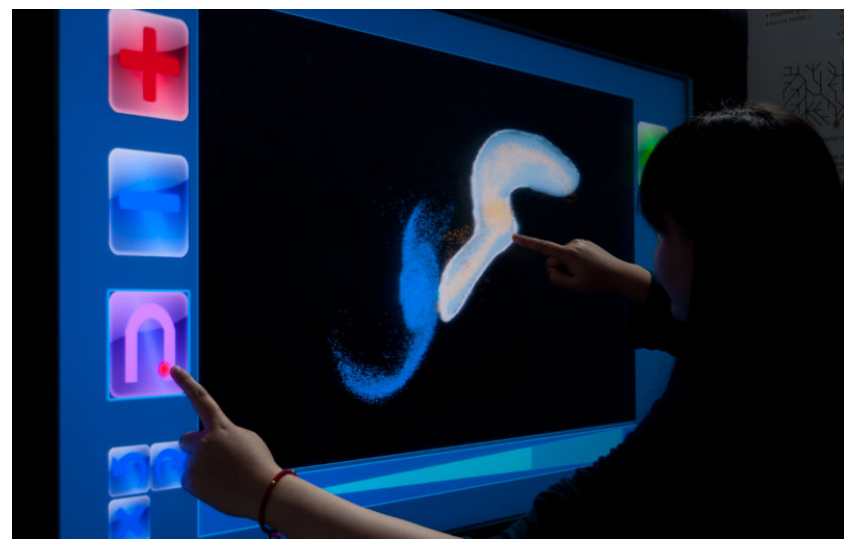

Fig. 1: Lasso-based selection interaction on the 2D touch surface to obtain a selection in 3D space [11].

collaborative and immersive environments as well as highperformance computing, to create new applications of visualization across disciplines, to develop suitable evaluation methodologies for the complex data exploration tasks, and to educate about natural interfaces for visualization and educate using natural interfaces for visualization.

\section{Challenge 1: New mappings for the fundamental visual data exploration tasks}

To accomplish most high-level data visualization and exploration tasks, any interactive system must support a range of low-level interactions, including:

- changing views/navigating 3D space,

- changing visualization styles/types,

- picking objects/locations in 3D,

- selecting sub-spaces, objects or groups of objects, visualization elements (e.g., flow/path lines),

- specifying 0D, 1D, 2D, and 3D locations/ranges in 3D space for operations such as seed particle placement,

- placing/manipulating cutting planes and other widgets,

- planning paths/interventions,

- selecting/adjusting well-defined views,

- generating data value read-outs/measurements, and

- specifying/manipulating many data exploration parameters (color scales, value ranges, etc.).

Standards have been established in PC/desktop computing environments for many of these low-level tasks, but new mappings are needed for our envisioned novel interactive platforms. When using touch input, for example, we not only have to deal with 2D-input-to-3D-output mapping [3] but also have to be aware of modality and precision issues. In contrast to a mouse-and-keyboard setting, touch input does not provide buttons or keys that could be used to change from one type of input modality to another, e. g. to change from rotation to zooming to panning when navigating the $3 \mathrm{D}$ space. Gestural forms of interactions might instead be used to specify interaction modalities. However, finding appropriate gestural interaction mappings is not as simple as it may sound. While the one-finger pan and two-finger rotate-scale-translate gestures are wellestablished and accepted, it is not clear how to find appropriate mappings for the remaining fundamental tasks. 
Moreover, in scientific visualization we often need not only to switch the interaction modality but also, and at the same time, specify parameters via direct manipulation. One possible solution to this is the use of interactive widgets in combination with (potentially bi-manual) gestural interaction. For example, a gestural interaction could generate different results depending on which part of the interaction widget is touched and/or in which direction an initial motion is performed. Essentially this means that interactions are being specified by means of springloaded modes: modes that are only maintained for as long as an interaction element is touched. Examples for such interactions have recently been explored for a number of the fundamental tasks including view navigation [1, 12], path planning [1] (Fig. 3), and selection [11].

When touch and other natural interfaces for scientific visualization are designed correctly, the new abilities they provide to the user can be impressive. Using touch-enabled displays for data selection creates the expectation that selection should be as simple as merely grasping objects in the real world. This is, of course, not technically possible when working with two-dimensional touch surfaces, but if we find ways for people to easily specify a 3D region using the 2D surface, we can achieve a powerful effect. CloudLasso [11] (Fig. 1) does this by solving the underconstrained problem via heuristics and giving users the impression of selecting exactly what they intended to select. Similarly, with Interactive Slice WIM [1], users move their fingers on a touch surface to quickly specify a wide range of shapes that are linked to 3D cutting planes to dynamically adjust complex selection volumes for bundles of fluid flow data; the result is not only useful for making volumetric selections, but because it is coupled so closely to the 3D visualization it also provides a new method for real-time data exploration that is immediately usable by any scientist.

An additional challenge of many emerging natural interface modalities is that they are often imprecise-finger tips have large surface areas, certainly larger than one pixel (the "fat finger problem"). This fact is particularly troublesome for visualization applications as they often require high precision when controlling the interface. One possibility to overcome the precision issue is to provide a means to constrain the input by restricting the degrees of freedom, or by using dedicated display-gain ratios. This problem of precise, controlled, and constrained interaction remains largely unsolved for scientific visualization applications and is a critical area of focus within this research challenge.

Another critical research area is using touch input in stereoscopic scientific visualization environments. Since the dataset can be perceived in 3D space but input is only on a 2D touch surface, this can lead to the problem of "touching through" objects or bumping into a near-to-invisible touch surface. Moreover, as one fixates on an object in front or behind the touch surface, one perceives the touching finger to appear twice- the parallax problem. One possible solution is to use two surfaces, one for touch input and one for the stereoscopic data display [1] (Fig. 3), but additional and more portable solutions, such as creative intentional

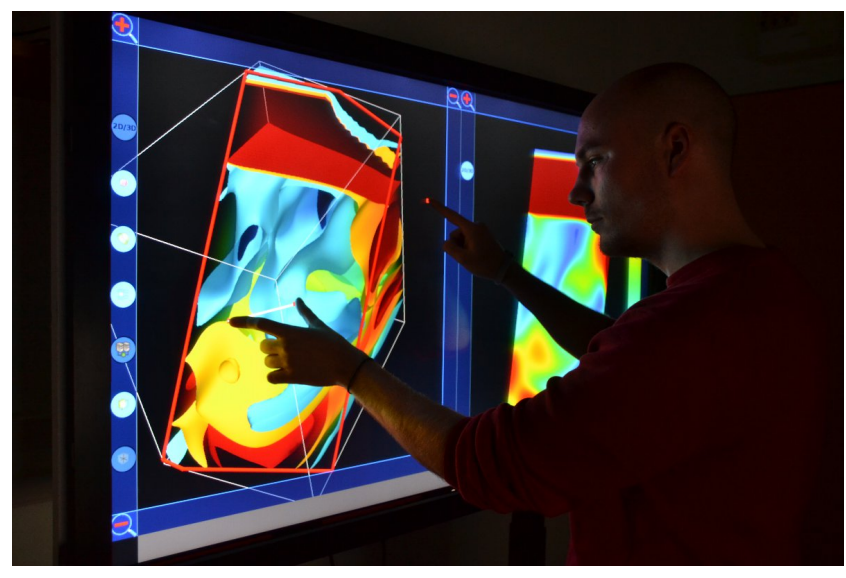

Fig. 2: Fluid flow exploration integrating a number of lowlevel exploration tasks [6] including navigation, cutting plane manipulation, seeding, data drilling, changing visualization techniques, and color scale interaction.

use of perceptual illusion or deformable touch-capable input devices, may also be possible.

\section{Challenge 2: Toolkits and theories of interaction}

Beyond identifying appropriate mappings for the fundamental low-level interactions needed for visualization applications, another major challenge is integrating these interaction techniques to also function naturally when used in combination as part of a complex interactive visualization tool/application. This means, of course, that the chosen interaction mappings for the individual low-level methods must not conflict with each other. Moreover, the same interaction technique may need to be used for interacting with different data types (e.g., manipulating both a 3D isosurface and raw 3D point cloud data).

Current cutting-edge research in this area has taken some initial steps toward addressing the problem, specifically within the contexts of fluid flow [6] (Fig. 2), medical imaging [1] (Fig. 3), and geophysical data visualization. Many of the approaches use a bi-manual or multi-finger input to specify the interaction type. Here, the non-dominant hand or a finger selects the mode based on the location within a widget, freeing up the dominant hand or another finger to perform the actual (precise) interaction/parameter control.

Building upon these emerging case studies, an important research question that must be addressed within the challenge is: Is there/can there be a theory of natural interactions for science in general that a molecular biologist/chemist can use just as an astronomer, just as a fluid dynamics researcher, just as a behavioral biologist? Related, to what extent can this theory or theories be distilled into a toolkit - a set of fundamental interaction tools that is applicable to a wide variety of scientific domains? Answering these questions will be imperative for the longevity and growth of natural interfaces for scientific visualization, but the topic is complex. It seems likely that some scientific domains may require specific, highly-tuned interfaces. If these lead to major differences across domains, then reconciling these in the interaction toolkits we create 


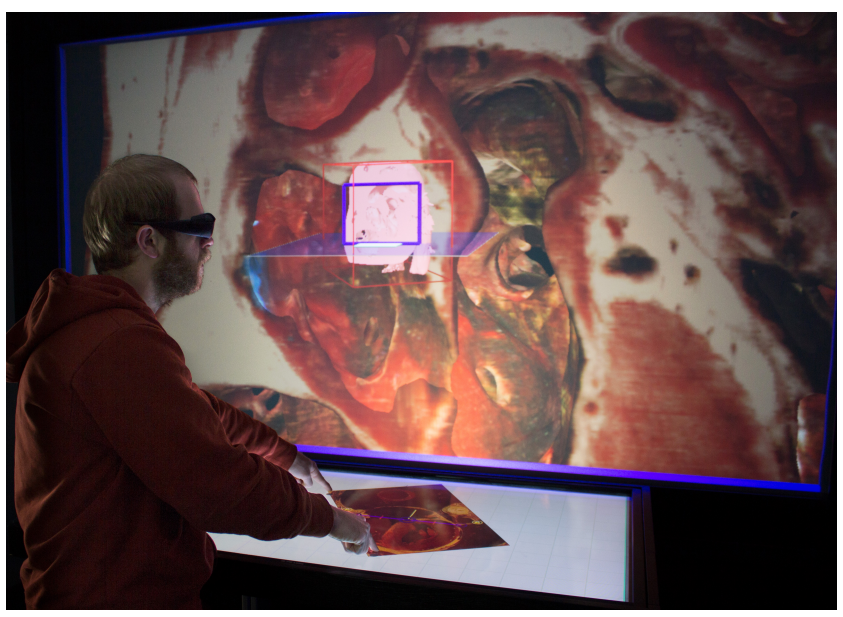

Fig. 3: A multi-touch based natural user interface is combined with 3D stereoscopic visualization [1].

(or expanding the number of toolkits we support) will be critical. Even if we just consider a single scientific domain, the question remains of whether a standard theory of the best use of natural interfaces in this domain along with an appropriate toolkit to support it can be developed. As we work toward these it will be important to be flexible, evolving our notion of best practices for natural interfaces as new technical capabilities become available that may again radically change our concept of what we consider to be a "natural interaction."

\section{Challenge 3: Collaboration, immersive environ- ments, and high-performance computing}

A next major challenge for the community will be scaling up the new techniques we develop to integrate with major visualization systems. An important focus will be developing new methods for using natural user interfaces coupled with visualization to work more effectively with highperformance computing (supercomputing) and with massive data repositories. Indeed, leveraging high-performance computing and working with big data is in general a major challenge for visualization research as a whole; we (re)emphasize the importance here because of the especially valuable role that we believe natural interfaces can play in addressing this challenge.

Consider, for example, the future role of visualization in simulation-based engineering. As HPC techniques continue to advance and provide new capabilities for more accurate and fast simulations of engineering problems, virtual prototyping will become increasingly important relative to traditional physically based bench-top design. Engineers will need to interpret massive amounts of multi-dimensional spatial and time-varying data and need to effectively control the processes that generate these data (e.g., spawning new simulation runs and parameter-space studies, setting boundary conditions, linking simulated data with other data repositories of material properties and more). If natural user interfaces could be scaled up to the point that they can interface with these high-end visualization problems, then the improvement in simulation-based engineering would likely be profound. In a simple example, an engineer might use a direct natural user interface to manipulate the shape of a 3D mechanism and/or, for a medical device, its position and orientation within a human anatomical model. This natural interaction could automatically spawn a series of high-end HPC calculations, the results of which would be fed back to the user via visualization to enable real-time multi-dimensional simulation-based design. Importantly, throughout this powerful process, the engineer's focus would be on the engineering design problem and on manipulating visualizations of the problem directly with his or her hands, not on details of the simulation setup or scripting.

As these new scaled-up interactive workflows and processes emerge, the environments in which we work with data will also need to scale. Whereas much of the current research in natural user interfaces focuses on handheld and desktop-scale surface devices, our most challenging data visualizations will likely require natural user interfaces that work at the scale of high-resolution powerwall displays, CAVE's, and immersive tables. Fig. 3 shows the Minnesota 3D Touch Workbench, a mid-scale environment that supports head-tracked stereoscopic visualization together with multi-touch input [1]. One of the advantages of this environment relative to a traditional immersive platform, such as the CAVE, is that the 2D touch display can serve as a workspace for traditional 2D data operations (e.g., managing datasets, annotating data, setting up simulations), which are typically essential to doing real science or engineering work, but are often difficult to perform or left out of fully immersive 3D environments. As in this example, research is needed not just in new interface techniques but also to develop new display environments or combine existing environments in new ways so that users gain the advantage of interactive immersive systems while retaining the abilities to manage, organize, annotate, and generally work with data in ways that have traditionally been successful in desktop-based systems.

An essential final area of focus within the scaling up challenge is supporting collaboration. Useful collaborative visualization can take many forms (e.g., co-located vs. distributed, synchronous vs. asynchronous) and each of these is important to investigate within the broader context of research on collaborative visualization [2]. Within immersive environments, co-located synchronous collaboration is often observed; for example, the systems pictured in Fig. 2 and 3 were not built primarily to support collaboration, but this is one of the most regularly cited benefits of the systems by users. The future research agenda for natural user interfaces in visualization should specifically target supporting and evaluating collaboration in these environments. For example, we believe natural user interfaces could support a variety of useful methods for transitioning between individual and team work, which could powerfully improve the effectiveness of data visualizations. 

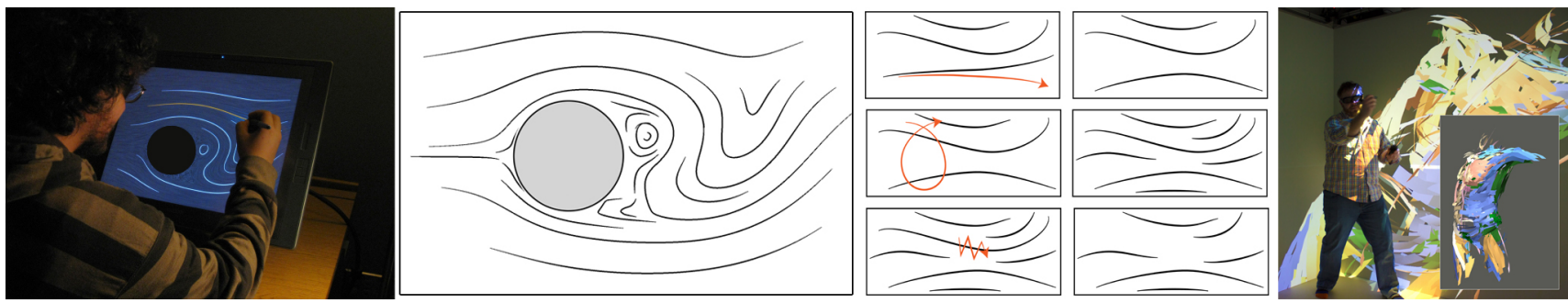

Fig. 4: Two natural user interfaces that enable traditionally trained artists to work with scientific visualization. The left and middle images depict a sketch-based interface for stylistic illustration of 2D vector fields [9]. The rightmost image shows a virtual reality 3D sketching tool used for both art practice and prototyping scientific visualizations [5].

\section{Challenge 4: New applications across disciplines}

The next important research challenge is to demonstrate and understand how natural user interfaces can make even extremely complex visualization systems more broadly accessible. For scientists and engineers, our goal should be to enable these users to leverage the most powerful data-driven computer graphics methodologies developed in our community without needing to learn to program or script new workflows, or even to navigate the complex hierarchical menus and visual programming languages that are used in many of today's desktop visualization tools. In practice, current interfaces often create roadblocks to use; we envision, in contrast, that cutting-edge research combining natural user interfaces with visualization can enable new interaction styles for working with data that are fluid and seamless, similar to what we see now with handheld devices and touch interfaces for non-scientific applications. This would certainly amplify the impact of visualization research across many science and engineering disciplines, making many new applications possible through wider tool use.

Perhaps even more exciting than increasing the impact of visualization in fields that already have some experience with its benefits is the potential to open up completely new application areas. Fig. 4 demonstrates two recent concrete examples. The system pictured on the left enables traditionally trained illustrators and graphic designers to use pen-based input to sketch stylized hand-drawn renderings of flow patterns [9]. The interface intelligently interprets each drawn stroke based on constraints implied by the underlying fluid flow dataset. The system thus combines artistic design decisions made by a trained graphic designer together with underlying data to produce accurate stylized hand-drawn renderings of flow patterns. The rightmost image portrays a 3D tool for creating virtual sculpture by "painting in the air," used both for art practice and as a valuable sketchpad for prototyping 3D scientific visualizations [5]. Due specifically to the natural user interfaces employed, both of these systems have enabled artists and designers trained in visual depiction to work creatively on scientific visualization problems using the latest computer graphics technologies and without any knowledge of programming. Further research in this area can engage additional nontraditional scientific visualization users and practitioners.

\section{Challenge 5: Evaluation}

As new natural user interfaces and applications are developed, expanded, and refined over time, it will be important that these efforts are closely coordinated with research into novel approaches to evaluating scientific visualizations. Low-level performance measures as regularly utilized in the HCI community are often so far removed from realistic scientific visualization tasks that their utility is limited. On the other hand, the combined visuals and interfaces that result from this research are so new and different than what domain experts have seen before that it can be difficult to perform unbiased expert-based case study evaluations. It will be essential to develop and disseminate new methods, guidelines, and success stories for evaluations (see also [3]).

\section{Challenge 6: Education}

Natural interfaces can have a major impact on education in our community and beyond. We highlight two complementary areas of focus within this challenge.

- Educate visualization users and practitioners about natural user interfaces.

- Leverage compelling natural interfaces for visualization as teaching tools for use in the sciences and other domains.

In scientific visualizations the user needs to understand the mapping from data to visual form to work effectively; the process of becoming familiar with how to create and read these mappings is often called visual literacy-it is a skill that needs to be taught and learned. Similarly, we need to teach users and practitioners about how to work most effectively with scientific visualizations that enable new interactive processes and experiences. Ben Shneiderman's mantra, "overview first, zoom and filter, then details-ondemand" is one useful guideline in this direction since it emphasizes a process of visualization that evolves over time and necessarily involves user interaction. In his book "Sketching User Experiences: Getting the Design Right and the Right Design," Bill Buxton also emphasizes the need to design computer tools based on the interactive experiences they enable. One way that we can teach this is by increasing the emphasis on the experience of visualization within our teaching. For example, following Buxton's notion of "sketching" user interfaces, students in a recent visualization course at the University of Minnesota learned not only traditional computer graphics and data-driven visualization 
techniques but also how to design visualizations that incorporate novel natural user interfaces. Specific class assignments included building physical prototypes of natural user interfaces using paper, tape, scraps of metal and wood, flashlights, and other physical props.

We also recognize the important role that natural scientific visualization interfaces can play in education within the sciences and other domains simply because these interfaces make data visualization accessible to so many people. In chemistry and physics, students will be able to perform hands-on experiments using natural interfaces coupled with 3D scientific visualizations. Many other domains are also natural fits for new educational tools. Today, there are several innovative natural interfaces for education, for example, in chemistry and math [7], but the data visualization aspects of these tools are quite limited compared to the current state of the art in scientific visualization. What we call for in the future is a truly integrated research effort that simultaneously advances both natural user interface research and visualization based on 3D computer graphics. If we can address these topics in tandem, then the realism, accessibility, and ultimate effectiveness of educational visualization systems can be increased dramatically.

\section{Conclusions}

Together these specific challenges define a research agenda that we believe will enable a paradigm shift in scientific visualization, transforming both the power and the accessibility of the visualization systems we develop. We urge the community to start by focusing on Challenge 1 to lay a foundation for using natural interfaces to accomplish dataintensive scientific tasks, many of which have until now been considered too complex to address using natural user interfaces. Based on this new set of techniques, our aim should be to establish the fundamental theories and toolkits of natural interfaces for science described in Challenge 2. As this basic research continues, it will enable the type of paradigm changing advances we highlight in Challenge 3. In the longer run, we believe natural interfaces coupled with simulation and other forms of high-performance computing will transform the way that we do engineering and design; our community can lead that and other similar transformations, but we need to make the advanced visualization tools that we develop work for people-this is the critical role we envision for natural user interfaces. Along the way, we see an always increasing opportunity to engage with new applications, and, in particular, with new user bases not only in the sciences, but also in the arts, the humanities, and other disciplines, as discussed in Challenge 4 . All these advances will demand new evaluation methodologies described in Challenge 5. Similarly, the educational initiatives we outline in Challenge 6 must occur in parallel with the other research activities. There is great potential for impact from education not only within our own scientific communities but also as we embrace opportunities for outreach and new applications in K-12 and higher education.

Today, interest in and awareness of natural user interfaces is higher than ever before. We can thank the many recent commercial efforts as well as the research efforts that preceded them for the technologies that enable this new paradigm of computing. The challenge for the scientific visualization community is to learn how best to leverage these and related future technologies. We argue strongly that this challenge is not just a simple application of known techniques to visualization, but rather a core research topic including significant domain-specific technical and algorithmic challenges that are of fundamental importance to the future of scientific visualization. Our hope is that the specific challenges outlined in this article can serve as part of a call to action on cutting-edge research on natural user interfaces for scientific visualization.

\section{ACKNOWLEDGMENTS}

Thanks to our students and collaborators. Keefe's work was supported in part by grants from the NSF (CAREER IIS1054783 and IIS-1218058) and by the National Academies Keck Futures Initiative. Isenberg's work was supported in part by a French DIGITEO chair of excellence.

\section{REFERENCES}

[1] D. Coffey, N. Malbraaten, T. Le, I. Borazjani, F. Sotiropoulos, A. G Erdman, and D. F. Keefe. Interactive Slice WIM: Navigating and Interrogating Volume Datasets Using a Multi-Surface, Multi-Touch VR Interface. TVCG, 18(10):1614-1626, 2012. doi > 10.1109/ TVCG.2011.283

[2] P. Isenberg, N. Elmqvist, J. Scholtz, D. Cernea, K.-L. Ma, and H. Hagen. Collaborative Visualization: Definition, Challenges, and Research Agenda. Information Visualization, 10(4):310-326, 2011. doi $>10.1177 / 1473871611412817$

[3] P. Isenberg, T. Isenberg, T. Hesselmann, B. Lee, U. von Zadow, and A. Tang. Data Visualization on Interactive Surfaces: A Research Agenda. $C G \& A, 33(2), 2013$. To appear.

[4] D. F. Keefe. Integrating Visualization and Interaction Research to Improve Scientific Workflows. $C G \& A, 30(2): 8-13,2010$. doi $>10$ 1109/MCG.2010.30

[5] D. F. Keefe, D. Acevedo, J. Miles, F. Drury, S. M. Swartz, and D. H. Laidlaw. Scientific Sketching for Collaborative VR Visualization Design. TVCG, 14(4):835-847, 2008. doi> 10.1109/TVCG.2008.31

[6] T. Klein, F. Guéniat, L. Pastur, F. Vernier, and T. Isenberg. A Design Study of Direct-Touch Interaction for Exploratory 3D Scientific Visualization. CGF, 31(3):1225-1234, 2012. doi > 10.1111/j.1467 $-8659.2012 .03115 . \mathrm{x}$

[7] J. J. LaViola, Jr. and R. C. Zeleznik. MathPad2: A System for the Creation and Exploration of Mathematical Sketches. TOG 23(3):432-440, 2004. doi> 10.1145/1015706.1015741

[8] B. Lee, P. Isenberg, N. H. Riche, and S. Carpendale. Beyond Mouse and Keyboard: Expanding Design Considerations for Information Visualization Interactions. TVCG, 18(12):2689-2698, 2012. doi> 10.1109/TVCG.2012.204

[9] D. Schroeder, D. Coffey, and D. Keefe. Drawing with the Flow: A Sketch-Based Interface for Illustrative Visualization of 2D Vector Fields. In Proc. SBIM, pp. 49-56. Eurographics, 2010. doi $>10$. 2312/SBM/SBM10/049-056

[10] D. Wigdor and D. Wixon. Brave NUI World: Designing Natural User Interfaces for Touch and Gesture. Elsevier, Amsterdam, 2011.

[11] L. Yu, K. Efstathiou, P. Isenberg, and T. Isenberg. Efficient StructureAware Selection Techniques for 3D Point Cloud Visualizations with 2DOF Input. TVCG, 18(12):2245-2254, 2012. doi > 10.1109/TVCG 2012.217

[12] L. Yu, P. Svetachov, P. Isenberg, M. H. Everts, and T. Isenberg. FI3D: Direct-Touch Interaction for the Exploration of 3D Scientific Visualization Spaces. TVCG, 16(6):1613-1622, 2010. doi > 10.1109/ TVCG.2010.157 
Daniel F. Keefe is a McKnight Land-Grant Assistant Professor in the Department of Computer Science and Engineering at the University of Minnesota, where he directs the Interactive Visualization Lab. He specializes in visualization and interactive computer graphics. Keefe received a $\mathrm{PhD}$ in computer science from Brown University. $\mathrm{He}$ is a senior member of the IEEE and a member of the ACM. Contact him at keefe@cs.umn.edu.

Tobias Isenberg is a Senior Research Scientist at INRIA Saclay in France. He specializes in illustrative rendering and interactive visualization. Isenberg received his doctorate in computer science from the University of Magdeburg. He is a member of the IEEE, the ACM, ACM SIGGRAPH, and the Eurographics Association. Contact him at tobias.isenberg@inria.fr. 